\title{
Über die Gliederung des Quartärs und Pleistozäns
}

\author{
Von Paul Woldstedt, Bonn
}

Mit 1 Abbildung im Text

\begin{abstract}
$\mathrm{Z}$ u s a m m e n f a s s u $\mathrm{ng}$. Es werden Gründe dafür vorgebracht, daß das Holozän als besondere Epoche angesehen werden kann. Für das Pleistozän werden verschiedene Einteilungen vorgelegt, von denen die in Tab. 2 wiedergegebene als beste angesehen und zur Diskussion gestellt wird.

Im Anschluß daran wird die Frage einer weiteren Teilung sowohl der Mindel (=Elster)- als auch der Saale (=Riß)-Vereisung diskutiert. Für beides sprechen gute Gründe; doch fehlt noch eine sichere Bestätigung. Schließlich wird die Einteilung der Letzten Eiszeit (Würm, Weichsel) erörtert und eine etwas veränderte Gliederung vorgeschlagen.
\end{abstract}

$\mathrm{Sum}$ mary. There are reasons to take the Holocene as a separate epoch. For the Pleistocene several divisions are discussed. The division given in table 2 seems to be the best.

The question of a further division of the Mindel (=Elster) and the Riss (=Saale) Glaciations is discussed, especially that of a separate "Warthe Glaciation" (Riss II ?). There are good reasons for assuming longer intervals within both glaciations; but a definite answer to this question cannot be given so far. Lastly a new subdivision of the Last Glaciation (Würm = Weichsel) is proposed.

I.

Eine große Zahl von Forschern teilt das Quartär ein in Ple is to zä n und Holo$z$ ä n (früher sprach man im deutschen Schriftum auch von Diluvium und Alluvium). Die beiden Abschnitte sind, was ihre Dauer anbelangt, recht ungleich: das Holozän umfaßt nur rund 10000 Jahre, das Pleistozän wohl mindestens 1 Million, vielleicht sogar erheblich mehr. So schlagen einzelne Forscher, wie z. B. R. F. Flint (1957, S. 284) vor, das Holozän als besondere Epoche verschwinden zu lassen und das Ganze unter dem Begriff des Pleistozäns zusammenzufassen. Wenn man bedenkt, daß das Holozän offenbar nichts anderes ist als eine Interglazialzeit, die auf die Letzte Eiszeit folgt und der voraussichtlich eine neue Eiszeit folgen wird, dann scheint dieser Vorschlag durchaus berechtigt zu sein.

Aber dem steht ein anderer Gesichtspunkt entgegen. Das Holozän, das bis zur Gegenwart führt, ist der geologischen Erforschung in ganz anderer Weise zugänglich wie alle älteren Formationen. So ist hier eine Feingliederung möglich, wie sie sonst nicht durchgeführt werden kann. Das Studium des Holozäns geht über in das allgemeine Studium der geologischen Vorgänge (die sog. „Allgemeine Geologie“). Man denke nur an die Entwicklung der Küsten, an die Gletscher und Inlandeise, die vulkanischen Erscheinungen, die Tiefseeböden, die Vegetationsgeschichte (Pollenanalyse!) usw. Die genaue Behandlung dieser Dinge in der Gegenwart und jüngsten Vergangenheit ist in einem Maße möglich wie bei keiner älteren geologischen Formation. So ist die Behandlung des Holozäns als einer besonderen Epoche durchaus berechtigt, und ich schließe mich dem Kreise derer an, die das Holozän als besondere Abteilung bestehen lassen wollen. Es herrscht weitgehende Übereinstimmung darüber, daß der Beginn des Holozäns bei ungefähr 10000 Jahren vor heute anzusetzen ist.

Was nun das Ple is to z ä n anbelangt, so hat sich mit seiner weiteren Einteilung im Jahre 1932 der 2. Internationale Quartär-Kongreß in Leningrad beschäftigt. ${ }^{1}$ ) Damals

1) In den Kongreß-Berichten findet sich darüber allerdings keine Mitteilung. 
waren nur die vier alpinen (und nordamerikanischen) Eiszeiten bekannt, und es wurde folgende Einteilung vorgeschlagen:

\begin{tabular}{|c|c|}
\hline $\begin{array}{l}\text { Würm-Eiszeit } \\
\text { Riß/Würm-Interglazial }\left(\text { Eem²) }^{2}\right)\end{array}$ & $\begin{array}{l}\text { Jung- } \\
\text { Pleistozän }\end{array}$ \\
\hline $\begin{array}{l}\text { Riß-Eiszeit } \\
\quad \text { Mindel/Riß-Interglazialzeit (Holstein) }\end{array}$ & $\begin{array}{l}\text { Mittel- } \\
\text { Pleistozän }\end{array}$ \\
\hline $\begin{array}{l}\text { Mindel-Eiszeit } \\
\text { Günz/Mindel-Interglazialzeit (Cromer) } \\
\text { Günz-Eiszeit }\end{array}$ & $\begin{array}{l}\text { Alt- } \\
\text { Pleistozän }\end{array}$ \\
\hline
\end{tabular}

Eine vom Internationalen Geologenkongreß eingesetzte Kommission mit der Aufgabe, die Grenze Plio/Pleistozän festzulegen, kam $1948 \mathrm{zu}$ dem Ergebnis, daß diese Grenze dort zu legen sei, wo sich die erste weltweite, zu Vereisungen führende Abkühlung bemerkbar mache. Dies trifft zu für den Beginn des Calabriums in den marinen, für den Beginn des Villafranchiums in den kontinentalen Gebieten. Diese - an sich sehr langdauernden - Abschnitte sind also noch vor dem „Alt-Pleistozän“ einzuordnen. Man half sich zunächst, indem man dem Alt-Pleistozän ein „Altest-Pleistozän“ voranstellte (so auch in Woldstedt 1954).

Aber die Unterscheidung von „alt“ und „ältest“ ist nicht gut. Sie führt sehr leicht zu Verwechslungen und stimmt auch mit der Einteilung der anderen Formationen nicht überein, wo wir es fast überall mit „alt“, „mittel“ und „jung“ (oder ,unter“, „mittel“ und „ober“) zu tun haben. So bleibt nichts weiter übrig, als eine Neueinteilung des Pleistozäns in diese drei Abschnitte vorzunehmen. Wo aber sollen die Grenzen gezogen werden?

Es liegt nahe, den langen Abschnitt, der neu zum Pleistozän gekommen ist, das Calabrium bzw. Villafranchium im weiteren Sinne, als Alt-Pleistozän zu bezeichnen. Die Frage ist nur, wo die obere Grenze zu legen ist. H. L. Movius (1949) hat in einer ausführlichen Arbeit das ganze Villafranchium mit der Günz-Eiszeit parallelisiert. Das kann nun keinesfalls zutreffen. Nach neueren Untersuchungen sowohl in den Alpen (B. EBERL 1930, I. Schaeffer 1956, H. Graul 1949, S. Venzo 1956 u. a.) wie in den Niederlanden (W. H. Z AGWIJN 1960) liegen vor der Günz-Kaltzeit noch mehrere Warm- und Kaltzeiten, ehe wir in das Pliozän kommen. Sie alle zusammen bilden das Villafranchium. Dieses ist in Europa sowohl faunistisch wie floristisch charakterisiert durch tertiäre Relikte. In den Interglazialen, wie z. B. in Tegelen, Schwanheim usw., treten noch die tertiären Formen Carya, Pterocarya, Magnolia, Phellodendron usw. auf. Im Cromer-Interglazial sind diese Formen so gut wie völlig verschwunden, und wir finden zum ersten Male ein typisch quartäres Pollendiagramm (vgl. z. B. Cromer oder Bilshausen).

Ahnlich ist es mit der Fauna. Im Villafranchium treten in Mitteleuropa noch Formen wie Mastodon (Anancus) arvernensis u. dgl. auf. Auch diese sind im Cromer-Interglazial in Mittel- und Westeuropa verschwunden. Von den Elefanten sind im Villafranchium Elephas planifrons und E. meridionalis charakteristisch. Im Cromer-Interglazial entwickelt sich daneben Elephas antiquus, der von nun an die europäischen Interglaziale beherrscht. So liegt es nahe, in Europa das Altpleistozän mit der Günz-Eiszeit aufhören zu lassen und mit dem Cromer-Interglazial das Mittel-Pleistozän zu beginnen.

Schwierig ist es, eine geeignete Grenze zwischen Mittel- und Jung-Pleistozän zu ziehen. Mit dem Beginn der Riß-Eiszeit tritt in Mitteleuropa der echte Elephas primigenius auf, und man könnte denken, dies als Grenze zwischen Mittel- und Jung-Pleistozän zu benutzen. Ich habe dies im 2. Bande meines „Eiszeitalters" (1958) getan. Die dadurch erhaltene Einteilung wird in Tab. 1 wiedergegeben.

2) Die Bezeichnungen Eem, Holstein und Cromer sind jünger. 


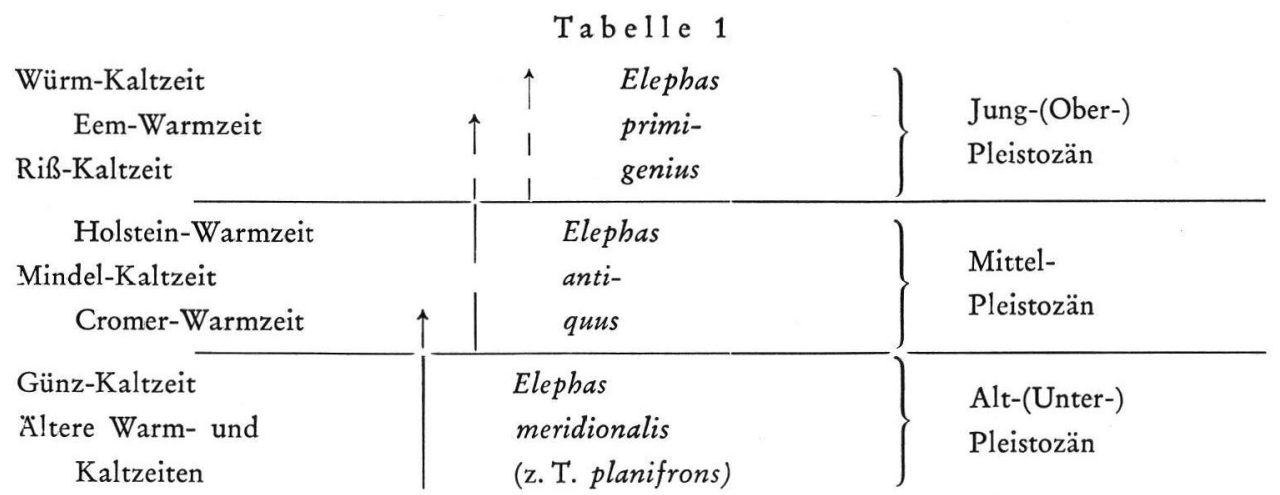

Es fragt sich aber doch, ob die hier angenommene Grenze zwischen Mittel- und Jungpleistozän auf allseitige Zustimmung rechnen kann. Denn in der ausländischen Literatur (vgl. z. B. die englische und französische Literatur über Afrika) wird meist überhaupt nur die Letzte Eiszeit (Würm) als Jungpleistozän bezeichnet, während die vorhergehende Warmzeit (Eem) noch zum Mittel-Pleistozän gerechnet wird. In mündlichen Diskussionen über diese Frage hat H. B. S. Cooke (damals Johannesburg, Südafrika) vorgeschlagen, als Jungpleistozän die Letzte Interglazialzeit (Eem) und die Letzte Eiszeit (Würm) zusammenzufassen, die Riß-Eiszeit dagegen noch zum Mittel-Pleistozän zu rechnen.

Die Grenze Mittel-/Jung-Pleistozän würde dann dort verbleiben, wo sie der InquaKongreß 1932 hingelegt hat, und es würde sowohl das Mittel- wie das Jung-Pleistozän mit einer Interglazialzeit beginnen. Die so abgegrenzten Zeiträume sind zwar sehr verschieden lang - und zwar sind sie umso länger, je älter sie sind - aber das entspricht etwa der perspektivischen Verkürzung, unter der wir sie sehen.

Diese eben skizzierte Gliederung ist in Tab. 2 wiedergegeben, wobei die (roh geschätzte) Dauer der einzelnen Abschnitte eingesetzt worden ist. Mir scheint heute diese Einteilung die geeignetste $\mathrm{zu}$ sein.

\section{Tabelle 2}

Holozän

$10000 \mathrm{~J}$.

\begin{tabular}{|c|c|c|}
\hline $\begin{array}{l}\text { Würm-Kaltzeit } \\
\text { Eem-Warmzeit }\end{array}$ & Jung-Pleistozän & $100-120000 \mathrm{~J} . ?$ \\
\hline $\begin{array}{l}\text { Riß-Kaltzeit } \\
\text { Holstein-Warmzeit }\end{array}$ & \multirow{2}{*}{ Mittel-Pleistozän } & \multirow{2}{*}{$300-400000 \mathrm{~J} . ?$} \\
\hline $\begin{array}{l}\text { Mindel-Kaltzeit } \\
\quad \text { Cromer-Warmzeit }\end{array}$ & & \\
\hline $\begin{array}{l}\text { Günz-Kaltzeit } \\
\text { Ältere Warm- und Kaltzeiten }\end{array}$ & Alt-Pleistozän & 1 Mill. J. ? \\
\hline
\end{tabular}

Zum Schluß sei noch kurz Stellung genommen zu dem Vorschlag von G. LÜTrIG (1959), der eine Reihe von neuen Stufennamen für das mitteleuropäische Pleistozän einführen möchte. Sein Vorschlag wird in Tab. 3 wiedergegeben.

Die Einteilung von G. Lütrıg beruht auf dem Begriff des Zyklus. Natürlich kann man diesen Begriff auch im Quartär verwenden $\left.{ }^{3}\right)$. Aber dann sollte man logischerweise den

3) G. Lürtrg (1958) hat solche Zyklen auch seiner Einteilung des italienischen P'eistozäns zugrunde gelegt. 
Tabelle 3

Einteilung des mitteleuropäischen Pleistozäns nach G. LütTig

\begin{tabular}{|c|c|c|}
\hline $\begin{array}{l}\text { Weichsel-Glazial } \\
\text { Eem-Interglazial }\end{array}$ & Utrecht-Stufe & Jung-Pleistozän \\
\hline $\begin{array}{l}\text { Saale-Glazial } \\
\text { Holstein-Interglazial }\end{array}$ & Oldenburg-Stufe & Mittel-Pleistozän \\
\hline $\begin{array}{l}\text { Elster-Glazial } \\
\quad \text { Cromer-Interglazial }\end{array}$ & Erfurt-Stufe & \\
\hline $\begin{array}{l}\text { Weybourne-Kaltzeit } \\
\text { Tegelen-Warmzeit } \\
\text { Butley-Kaltzeit }\end{array}$ & Aachen-Stufe & Alt-Pleistozän \\
\hline
\end{tabular}

Zyklus jedesmal mit einer Kaltzeit beginnen - denn die ganze Formation des Quartärs fing mit einer Kaltzeit an - und mit dem Ende der darauf folgenden Warmzeit aufhören lassen. Der letzte Zyklus würde dann mit der Letzten Eiszeit (Würm) beginnen, und das — noch nicht beendete - Holozän wäre die zweite Hälfte dieses letzten Zyklus.

Es entsteht aber die Frage, ob die Einführung von Zyklen mit den dazu notwendigen neuen Namen für das Quartär wirklich einen Gewinn darstellt.

In Afrika ist man meines Erachtens mit der Einführung solcher Stufen-Bezeichnungen nicht sehr glücklich gewesen. Dort sollten nach den Entschließungen des 3. Panafrikanischen Kongresses in Livingstone (1957) nur die Stufen-Bezeichnungen Kageran, Kamasian, Kanjeran und Gamblian verwendet werden, wobei jede dieser Stufen (mit Ausnahme der letzten) eine Pluvialzeit und die darauf folgende Interpluvialzeit umfassen sollte. Mit zunehmender Kenntnis erweist es sich aber immer mehr als notwendig, dann noch hinzuzufügen, ob es sich im einzelnen Falle um die Pluvial- oder die Trockenzeit der betreffenden Stufe handelt. Es wird dann etwa von der „Kamasian/KanjeranInterpluvialzeit" usw. gesprochen.

Zweifellos wäre es hier besser gewesen, die obengenannten Bezeichnungen auf die Pluviale zu beschränken und für die Interpluviale besondere Namen einzuführen, so wie es in Europa und Nordamerika üblich ist. Denn, um nun auf die für Mitteleuropa vorgeschlagene Stufen-Einteilung zurück zu kommen: die erste entscheidende Frage bei jedem Vorkommen bleibt doch immer die: liegt es in einer Warm- oder in einer Kaltzeit? Das ist nicht nur wichtig für die Beurteilung der Faunen und Floren, sondern ebenso für die Lage des Meeresspiegels, für das Klima usw. Die zweite Frage ist die: wo liegt der Fundpunkt oder das Profil in der großen Einteilung: d. h. sind sie alt-, mittel- oder jungpleistozänen Alters? Die $Z_{w}$ ischenschaltung von Stufenbezeichnungen bringt dabei nichts Neues, Entscheidendes hinzu und ist insofern unnötig.

II.

Kurz sei hier noch auf die Frage einer weiteren Untergliederung von einzelnen Teilen des Pleistozäns eingegangen. Die nordische E ls t e r-E is z e it (=Mindel) soll nach den Untersuchungen von WEST \& Donner (1956) im östlichen England durch das „CortonInterstadial“ zweigeteilt sein. Befinden sich aber die von D. F. W. BADEN-Powell (1950) beschriebenen "Corton Beds" an ihrer ursprünglichen Lagerstätte (in ca. $30 \mathrm{~m}$ ü.d.M.,), dann werden wir eher an eine interglaziale und nicht interstadiale Bildung denken müssen. Denn in einem Interstadial können wir einen so hohen Ozeanspiegel nicht erwarten. Auch die Terrassenfolge im mittleren und unteren Rheingebiet spricht für eine Zweiteilung der Elster- oder Mindel-Eiszeit. Dieser gehören die Obere und die Mittlere Mittelterrasse an. Beide zeigen einen eigenen Schotterkörper und sind durch einen beträchtlichen Zeitraum der Erosion voneinander getrennt. Auch hier könnte man eher an ein 
Interglazial als ein Interstadial denken. In diesen Zeitraum wird von F. E. ZEUNER (1959), B. KurtéN (1960) und anderen das bekannte Vorkommen von Mauer (mit Hippopotamus!) gestellt.

Lange schon wird die Frage einer $Z$ we iteilung der Riß - oder S a a le-E is z e it (im weiteren Sinne) diskutiert. Als ich 1927 den Begriff der "Warthe-Vereisung“ aufstellte, dachte ich zunächst an eine stärkere Trennung dieser "Warthe-Vereisung " von der Saale-Vereisung, ließ aber die Frage zunächst offen, welcher Charakter dem Zeitraum zwischen Warthe- und Weichsel-Vereisung zukomme. Es zeigte sich später, daß hier ohne Zweifel die Eem-Interglazialzeit einzuordnen sei. Das war früher schon von A. JEsSEN u. a. (1918) wahrscheinlich gemacht worden, ergab sich aber später mit größerer Sicherheit (vgl. u. a. P. Woldstedt 1954a).

Was dann den Zwischenraum zwischen Saale und Warthe anbelangt, so glaubte ich 1929 hier nur ein Interstadial annehmen zu sollen. Demgegenüber betonte V. Milthers (zuletzt 1950) immer wieder, daß hier eine echte Interglazialzeit liegen müsse. Er folgerte dies aus der Verbreitung des Braunen Ostseeporphyrs, der im mittleren Norddeutschland und im angrenzenden Polen eine ausgeprägte Außengrenze zeigt. Nach Milthers muß das Eis in dem Z wischenraum Saale/Warthe mindestens bis nördlich der Ålandsinseln zurückgewichen sein. Vergleichen war das mit der entsprechenden Lage des Eisrandes in der Postglazialzeit, so muß in Mitteleuropa in dieser Phase bereits die Wärmezeit begonnen haben, d. h. wir hatten dort bereits ,interglaziale“ Verhältnisse. K. Picard (1960) und H. Stremme (1960) glauben, in Schleswig-Holstein interglaziale Böden gefunden zu haben, die in diese Zeit gehören sollen („Treene-Wärmezeit“). Schon früher hatte E. MücKenhausen (1939) betont, daß die Böden des "Warthe-Stadiums“ denen der Weichsel-Vereisung näher ständen als denen der Saale-Vereisung. Die Grenze legte er an die Hauptendmoränenzone der Lüneburger Heide (von den Schwarzen Bergen bei Harburg nach Südosten). Dieser Endmoränenzug stellt aber nicht die äußerste Ausdehnung der Warthe-Vereisung dar; sondern diese liegt, wie H. ILliEs (1955) nachwies, noch etwas weiter westlich bzw. südwestlich. So ist in diesem Bereich noch keine völlige Klarheit vorhanden.

Wenn zwischen Saale und Warthe eine echte Interglazialzeit vorhanden war, so sollten wir einen hohenMeeresspiegel erwarten - es sei denn, daß diese Interglazialzeit nur von sehr kurzer Dauer gewesen wäre. In der Postglazialzeit wurde das höchste „interglaziale“ Niveau erst am Ende des Atlantikums erreicht. Im Boreal lag der Meeresspiegel noch um $15-20 \mathrm{~m}$ tiefer. In einem Saale/Warthe-Interglazial müßten wir einen ähnlichen Verlauf des Meeresspiegel-Anstiegs erwarten. War dies Interglazial sehr kurz, dann ist vielleicht niemals ein hohes Meeresniveau erreicht worden; vielleicht lag das höchste erreichte Niveau un ter dem gegenwärtigen und ist deshalb bisher unserer Aufmerksamkeit entgangen. Handelte es sich aber bei dem Saale/Warthe-Interglazial um eine ebenso lange Warmzeit wie bei den anderen Interglazialzeiten, so müßten wir für diese ein höheres Meeresniveau (etwa zwischen Monastir II und Tyrrhen) erwarten.

$\mathrm{Daß}$ es sich bei dem Saale/Warthe-Interglazial um eine verhältnismäßig kurze Warmzeit gehandelt habe, könnte vielleicht aus der Terrassenfolge des Niederrheingebiets gefolgert werden. In dieser ist die "Warthe-Vereisung " zwar durch eine besondere Terrasse, die sog. „Krefelder Terrasse“, repräsentiert (vgl. u. a. W. PAAs 1961). Aber bei dieser handelt es sich offenbar um eine verhältnismäßig kurzfristige Bildung, die rheinaufwärts bisher nur bis in die Gegend von Bonn verfolgt werden konnte. Weiter oberhalb ist sie von der Unteren Mittelterrasse nicht mehr zu trennen. Während nun aber auf der Unteren Mittelterrasse eine kräftige Bodenbildung entwickelt ist, fehlt eine solche auf der Krefelder Mittelterrasse. Daraus folgert H. RemY (1960), daß diese kräftige Bodenbildung in die Saale/Warthe-Interglazialzeit gehöre.

Ein Pollendiagramm, das mit völliger Sicherheit in die Saale/Warthe-Interglazialzeit einzuordnen wäre, ist bisher nicht gefunden worden. Das von G. vON DER BRELIE (1955) 
als typisch für diese Interglazialzeit angesehene Profil von Oberohe und Neuohe („OheInterglazialzeit") soll nach R. HALLIK (1960) mit großer Wahrscheinlichkeit in die vorhergehende Holstein-Interglazialzeit gehören.

Fassen wir das über den Zeitraum zwischen Saale und Warthe Gesagte zusammen, so spricht zwar vieles für das Vorhandensein eines echten Interglazials an dieser Stelle, doch müssen eindeutige Beweise dafür noch erbracht werden. -

Wir kommen schließlich zur Frage einer weiteren Gliederung der Wü rm- oder W eichsel-E is z e it. Es besteht Einigkeit darüber, daß bei etwa 25-28000 J.v.h., d.h. vor dem bei etwa 18-20000 J.v.h. liegenden Würm-Maximum, ein Interstadial vorhanden war, das sog. "Paudorfer". Höchst überraschend ist dabei, daß diese Wärmeschwankung sich bis in die Gegend von Göteborg in Schweden ausgewirkt haben soll (vgl. F. BRotzen 1961). Aber die ausgeführten $C^{14}$-Bestimmungen lassen wohl kaum einen Zweifel daran, daß es sich hier tatsächlich um das Paudorfer Interstadial handelt.

A. Leroi-Gourhan (1960) gibt kurz vor dem Paudorfer ein weiteres Interstadial an, das A r c y - In te r s t a d i a 1, das zwar kurz, aber doch recht kräftig war. Offenbar haben diese beiden Interstadiale zusammen, die, wenn überhaupt, nur durch einen kurzen kälteren Abschnitt getrennt waren, doch ein stärkeres Rückschmelzen des Eises verursacht. Ein Eisfreiwerden der Gegend von Göteborg wäre aber nur verständlich, wenn vorher, d.h. im Mittelwürm, noch keine Verbindung zwischen dem Skandinavischen und dem Britischen Eis über die Nordsee herüber vorhanden war. Diese Verbindung war wahrscheinlich auf das Maximum im Jungwürm beschränkt. -

Im übrigen geht es hauptsächlich um die Frage des sog. „Göttweiger Interstadials", d. h. um die Frage, ob die Letzte Eiszeit durch ein länger dauerndes, verhältnismäßig warmes Interstadial oder gar einen 12-18000 Jahre dauernden "Interstadial-Komplex“ unterbrochen war, wie dies besonders H. Gross (zuletzt 1960) verfochten hat.

Als Stütze für das Vorhandensein dieses größeren "Göttweiger" Interstadials wurde das von Edith EBERS (1960) beschriebene „Interstadial von Hörmating “ angesehen. Für dieses lagen zwei C14-Datierungen vor: eines aus Torf ergab einen Wert von $45300 \pm$ 1000 J.v.h., ein anderes aus Holz einen solchen von mehr als 53000 J.v.h. H. Gross (1960) wählte den ersteren, um damit seinen "Göttweiger-Interstadial-Komplex “ zu stützen. Es ist aber die Frage, ob nicht der aus Holz gewonnene Wert von $>53000 \mathrm{~J}$. vorzuziehen ist. Dann aber käme entweder das Brörup-Interstadial oder das Eem-Interglazial in Frage. K. Brunnacker (1962) kommt in einer neuen Untersuchung zu dem Ergebnis, daß es ein „Hörmatinger Interstadial“" nicht gebe, sondern daß die als solche beschriebenen Ablagerungen in das Eem-Interglazial gehörten. Auf jeden Fall kann Hörmating nicht als eindeutiger Beweis für ein "Göttweiger Interstadial“ angesehen werden.

Was nun den Altwürm-Abschnitt anbelangt. so hat Helga REICH (1953) in ihrer Arbeit über die Interglaziale von Großweil und Pfefferbichl zuerst hingewiesen auf starke klimatische Schwankungen, die den Beginn der Würm-Eiszeit kennzeichnen. In ihren Pollendiagrammen kommen diese zum Ausdruck durch abwechselnde Dominanzen von Pinus und Picea. Dabei bedeutet das Auftreten der Pinus-Dominanz ein Herabrücken der Schneegrenze um rund $1000 \mathrm{~m}$ gegenüber der Laubwaldzeit des Interglazials; d. h. wir hatten schon recht bald im Altwürm nahezu volleiszeitliche Verhältnisse. Ahnliches haben dänische Untersuchungen (S. T. AnDERSEN 1961) für die bekannten Vorkommen Brörup und Rodebaek in Jütland ergeben. Hier waren bereits im Altwürm vegetationsarme Zeiten vorhanden, in denen Solifluktion herrschte. Schließlich zeigte sich dieselbe Entwicklung in den Niederlanden (nach Andersen, de Vries \& Z Zagwijn 1960). Nach den vorliegenden $\mathrm{C}^{14}$-Bestimmungen kann man mit großer Wahrscheinlichkeit folgern, daß die von H. REICH nachgewiesenen altwürmzeitlichen Abschnitte mit Picea-Dominanz dem Amersfoort- und dem Brörup-Interstadial entsprechen. 
Es entsteht die Frage, ob und in welcher Weise diese Schwankungen in den Lößprofilen registriert sind, und hier liegt es am nächsten, sie mit dem Abwechseln von Löß- und Schwarzerde-Phasen zu parallelisieren, das nach F. BrandtNER $(1954,1956)$ auf die „F-Wärmezeit" folgt und nach J. FINK (1956) den „Stillfrieder Komplex" charakterisiert. Während aber BRANDTNER in seiner „F-Wärmezeit" das "Göttweiger Interstadial“ sieht, parallelisiert J. FINK die Verlehmungszone an der Basis des Stillfrieder Komplexes mit dem Eem-Interglazial. Nach den heute vorliegenden C14-Bestimmungen (vgl. bes. KLímA \& KuKLA 1961) kann es kaum einem Zweifel unterliegen, daß J. FinK mit seiner Auffassung im Recht ist. ${ }^{4}$ )

Wir haben also die Schwarzerdephasen des Stillfrieder und ähnlicher „Komplexe“ zu parallelisieren mit den Interstadialen des Altwürms in Jütland (Rodebaek, Brörup) und in Holland (Amersfoort usw.) sowie mit den Waldperioden 11 und 13 (Picea-Dominanzen) im Profil Großweil-Ohlstedt von H. REICH (1953).

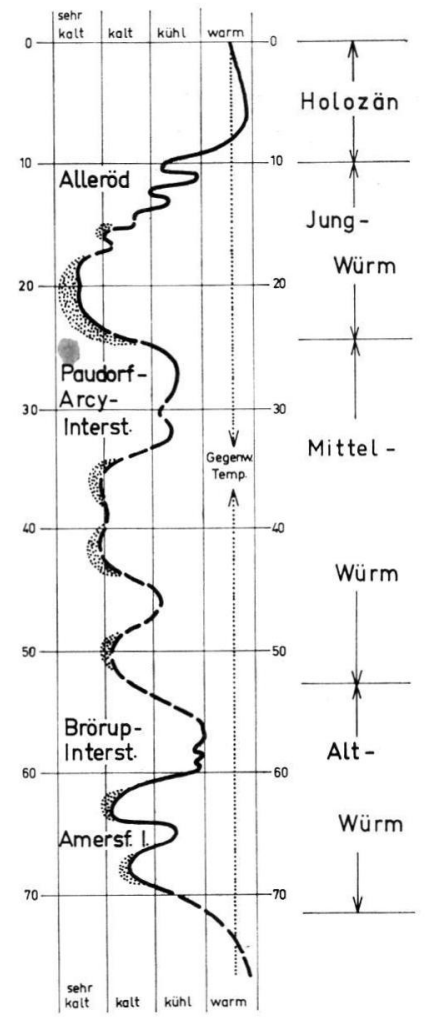
Abb. 1. Schematische Kurve, den (roh geschätzten) Temperatur- gang der Letzten Eiszeit ver- deutlichend. Punktiert: Haupt- zeiten der Lößbildung.

Es ergibt sich dabei eine wichtige Feststellung: Die Absenkung der Schneegrenze im Beginn von Würm geschah verhältnismäßig schnell. Sehr bald war, wie schon oben gesagt wurde, ein Betrag von ca. 1000 m erreicht. Dieser kam dem Maximalbetrag von ca. $1200 \mathrm{~m}$ schon recht nahe. Nach S. Th. Andersen (1961, S. 133) war schon im Abschnitt vor dem Rodebaek (=Amersfoort)-Interstadial die Juli-Temperatur um mindestens $10^{\circ} \mathrm{C}$ abgesenkt. In Jütland herrschte Solifluktion, und in einzelnen Gebieten begann bereits die Lößbildung. Wir können also die Klimakurve der Letzten Eiszeit nicht mit nur ganz

4) J. FinK hat mich kürzlich nochmals durch die wichtigsten Profile des österreichischen Lösses geführt, wofür ich ihm auch an dieser Stelle herzlichst danken möchte. 
geringen Schwankungen beginnen lassen, wie sie etwa in den Kurven von J. FiNK (1960) und $\mathrm{Hj}$. Müller-Beck (1959) gezeichnet worden sind, sondern müssen bereits im Altwürm mit einem ausgeprägt eiszeitlichen Klima (mit zwischengeschalteten interstadialen Schwankungen) rechnen.

Mit dem Ende des Brörup-Interstadials, d.h. bei rung 53000 Jahren vor heute, wird man am besten das Altwürm begrenzen — nicht mit dem sog. "Göttweiger Interstadial“, wie ich dies 1960 vorgeschlagen habe. Denn die Existenz eines solchen Interstadials ist inzwischen zweifelhaft geworden. Daß allerdings die Klimakurve zwischen 50000 und 30000 J.v.h. so gleichmäßig verlaufen sei, wie sie von Andersen, DE Vries \& Z AGwijn (1960) gezeichnet wurde, ist nicht sehr wahrscheinlich. Wir werden auch in diesem, als Mittelwürm zu bezeichnenden Abschnitt mit einzelnen wärmeren Schwankungen zu rechnen haben.

Wir kommen dann zum Interstadial Arcy-Paudorf, mit dem wir am besten den als Mittelwürm zu bezeichnenden Abschnitt enden lassen (wie schon 1960 vorgeschlagen). Es folgt der Jungwürm-Abschnitt (za. 25000 bis 10000 J.v.h.) mit dem Maximum der Eisausbreitung. -

Abb. 1 gibt eine schematische Kurve der Würm-Eiszeit in Mitteleuropa, die gegenüber der zuletzt (1960) von mir gegebenen etwas modifiziert ist. Es handelt sich um eine roh geschätzte Temperaturkurve, und zwar im wesentlichen eine der Juli-Temperaturen. Die früher von mir gegebenen Kurven waren z. T. solche des Eisrandes. Sie sind aber sehr viel hypothetischer, besonders für den älteren Teil der Würm-Eiszeit. Der Aufbau eines Inlandeises erfolgte sehr langsam und hinkte der Temperatur-Entwicklung, mindestens im Anfang, erheblich nach, worauf u. a. J. Büdel (1960) hingewiesen hat. -

Zum Schluß noch zwei Empfehlungen: 1. Der Ausdruck „Göttweiger Interstadial“, wie er besonders von H. Gross (1956), aber auch von mir selber $(1958,1960)$ verwendet worden ist, wird am besten nicht mehr angewandt. Denn die eine Gruppe von Forschern versteht darunter ein Würm-Inter s $\mathrm{tad}$ i a 1 , die andere ein Inter $\mathrm{g}$ a z i a 1 . Aber auch der Begriff: „Göttweiger Interglazial“ (G. GöTzINGER 1935) würde am besten verschwinden. Denn für das damit gemeinte Letzte Interglazial ist die eindeutige Bezeichnung „Eem“ vorhanden.

2. Ein zweiter Begriff, den die Quartärgeologen besser nicht verwenden sollten, ist der des Bodenkomplexes. Dieser von Bodenkundlern neuerdings viel angewandte Begriff (auch unter der Bezeichnung „Pedokomplex“) ist in der Quartär-Stratigraphie nicht brauchbar; denn er faßt Abschnitte zusammen, die stratigraphisch oft aufs schärf te zu trennen sind. Der Stillfrieder „Komplex“ z. B. umfaßt die Verlehmungszone der EemInterglazialzeit und einen großen Teil des Altwürms; d. h. eine der wichtigsten stratigraphischen Grenzen des Jungpleistozäns, die zwischen der Eem-Interglazialzeit und der Würm-Eiszeit, verläuft in der Mitte dieses Komplexes. Die Anwendung des Begriffes „Bodenkomplex“ birgt die Gefahr in sich, wichtige stratigraphische Grenzen zu übersehen.

Die hier vorgebrachten Anschauungen sind als Diskussions-Vorschläge gedacht. Ich bitte um Äußerungen dazu und werde diese gern in "Eiszeitalter und Gegenwart" abdrucken. Vielleicht kommen wir so zu einer Einigung in der Frage der Gliederung des Quartärs.

\section{$\mathrm{Sch}$ riften $-\mathrm{N}$ a chweis}

Andersen, Svend Th.: Vegetation and its environment in Denmark in the Early Weichselian Glacial (Last Glacial). - Danmarks geol. Unders., II. R. Nr. 75, 175 S., 15 Taf., Kopenhagen 1961.

Andersen, S. T., DE Vries, H., \& Zagwijn, W. H.: Climatic change and radiocarbon dating in the Weichselian Glacial of Denmark and the Netherlands. - Geol. en Mijnbouw 39, 38-42, Leiden 1960. 
Baden-Powell, D. F. W.: Field meeting in the Lowestoft district. - Proc. Geol. Ass. 61, 191-197, 1950.

BrandtNer, F.: Jungpleistozäner Löß und fossile Böden in Niederösterreich. - Dieses Jb. 4/5, 49-82, Ohringen 1954. - Lößstratigraphie und paläolithische Kulturabfolge in Niederösterreich und in den angrenzenden Gebieten. - Ebendort 7, 127-175, 1956.

von der Brelie, G.: Die Pollenstratigraphie im jüngeren Pleistozän. - Dieses Jb. 6, 25-38, 1955.

Brotzen, F.: An interstadial (radiocarbon dated) and the substages of the Last Glaciation in Sweden. - Geol. Fören. Forh. 83, 144-150, Stockholm 1961.

Brunnacker, K.: Bemerkungen zum Profil Hörmating/Obb. - Dieses Jb. 13 (im Druck).

BüDEL, J.: Die Gliederung der Würmkaltzeit. - Würzburger geogr. Arb., Heft 8, 45 S., Würzburg 1960.

EberL, B.: Die Eiszeitenfolge im nördlichen Alpenvorland usw. - Augsburg 1930.

EBERs, Edith: Drumlinkerne, ältere Würmschotter und das Würm-Interstadial-Profil von Hörmating/Obb. - Dieses Jb. 11, 64-76, 1960.

FINK, J.: Zur Korrelation der Terrassen und Lösse in Ósterreich. - Dieses Jb. 7, 49-77, 1956. - Leitlinien einer österreichischen Quartärstratigraphie. - Mitt. geol. Ges. Wien 53, 249-266, Wien 1960.

Götzinger, G.: Zur Gliederung des Lösses. - Verh. geol. Bundesanst. 1935, S. 126-132, Wien 1935.

Graul, H.: Zur Gliederung des Altdiluviums zwischen Wertach-Lech und Flossach-Mindel. - Natf. Ges. Augsburg 1949, 3-31.

Gross, H.: Das Göttweiger Interstadial, ein zweiter Leithorizont der letzten Vereisung. - Dieses Jb. 7, S. 87-101, 1956. - - Die Bedeutung des Göttweiger Interstadials im Ablauf der Würm-Eiszeit. - Ebendort 11, 99-106, 1960.

Hallik, R.: Die Vegetationsentwicklung der Holstein-Warmzeit in Nordwestdeutschland. - Z. deutsch. geol. Ges. 112, 326-333, Hannover 1960.

Illies, H.: Die Vereisungsgrenzen in der weiteren Umgebung Hamburgs, ihre Kartierung und stratigraphische Bewertung. - Mitt. geogr. Ges. Hamburg 51, 7-54, 1955.

Jessen, A., Madsen, V., Milthers, V., Nordmann, V.: Brörup Mosernes Lejringsforhold. - Danmarks geol. Unders., 4. R., Bd. 1, Nr. 9, Kopenhagen 1918.

Klima, B., KuкLA, J.: Absolute chronological data of Czechoslovak Pleistocene. - Quaternary of Central and Eastern Europe. Instyt. geol., Prace 34, 171-174, Warschau 1961.

Kurtén, B.: Chronology and faunal evolution of the earlier European glaciations. - Soc. Sci. Fennica, Comment. biol. 21, Nr. 5, 62 S., Helsingfors 1960.

Leroi-Gourhan, Arlette: Flores et climats du Paléolithique Récent. - C.r. Congr. préhist. France Monaco 1959, S.A. 6 S., Le Mans 1960.

LÜTTIG, G.: Stratigraphische Bemerkungen zum nichtmarinen Quartär Mittelitaliens. - Geol. Jb. 75, S. 651-662, Hannover 1958. - - Eiszeit - Stadium - Phase - Staffel. Eine nomenklatorische Betrachtung. - Ebendort 76, S. 235-260, Hannover 1959.

Milthers, V.: Die Gliederung und Verbreitung der skandinavischen Vereisungen in Nordwesteuropa. - Geol. Fören. Förh. 72, 257-268, Stockholm 1950.

Movius, H. L.: Villafranchian Stratigraphy in Southern and Southwestern Europe. - Journ. Geol. 57, 380-412, Chicago 1949.

MüCKenhausen, E.: Die Böden des Warthestadiums in Nordhannover. - Abh. naturw. Ver. Bremen 31, 335-346, 1939.

MülLER-BECK, Hj.: Bemerkungen zur Stratigraphie des mitteleuropäischen Jungpleistozäns. Dieses Jb. 10, 144-160, 1959

PAAS, W.: Rezente und fossile Böden auf niederrheinischen Terrassen und deren Deckschichten. Dieses Jb. 12, 165-230, 1962.

PiCARD, K.: Zur Untergliederung der Saalevereisung im Westen Schleswig-Holsteins. - Z. deutsch. geol. Ges. 112, 316-325, Hannover 1960.

Reich, Helga: Die Vegetationsentwicklung der Interglaziale von Großweil-Ohlstadt und Pfefferbichl im Bayrischen Alpenvor!and. - Flora 140, 386-443, Jena 1953.

Remy, H.: Der Löß am unteren Mittel- und Niederrhein. - Dieses Jb. 11, 107-120, 1960.

SCHAEFER, J.: Sur la division du Quaternaire dans l'avant-pays des Alpes en Allemagne. - Actes IV. Congr. intern. Quat., vol. II, 910-914, Rom 1956.

Stremme, H. E.: Bodenbildung auf Geschiebelehmen verschiedenen Alters in Schleswig-Holstein. Z. deutsch. geol. Ges. 112, 299-308, Hannover 1960. 
Venzo, S.: Stadi della glaciazione del "Donau“ sotto al Günz ed al Mindel nella serie lacustre di Leffe (Bergamo). - Actes IV. Congr. intern. Quat., vol. I, 65-85, Rom 1956.

West, R. G., \& Donner, J. J.: The glaciations of East Anglia and the East Midlands. - Quart. J. geol. Soc. London 112, 69-91, 1956.

Woldstedt, Paul: Das Eiszeitalter. Grundlinien einer Geologie des Quartärs. Bd. I, Stuttgart 1954. - - Dasselbe, Bd. II, Stuttgart 1958. - - Saaleeiszeit, Warthestadium und Weichseleiszeit in Norddeutschland. - Dieses Jb. 4/5, S. 34-48, 1954 [1954a]. - - Die Letzte Eiszeit in Nordamerika und Europa. - Ebendort 11, 148-165, 1960.

ZaGwiJn, W. H.: Aspects of the Pliocene and Early Pleistocene Vegetation in the Netherlands. Proefschr. Leiden, 78 S., 18 Taf., Maastricht 1960.

Zeuner, F. E.: The Pleistocene Period. - 2d. ed. London 1959.

Manuskr. eingeg. 29. 3. 1962

Anschrift des Verf.: Prof. Dr. Paul Woldstedt, Bonn, Argelanderstraße 118. 